\title{
Characteristics and Temperature Compensation of Non-Dispersive Infrared (NDIR) Alcohol Gas Sensors According to Incident Light Intensity
}

\author{
Humaira Hussain ${ }^{1}$, JinHo Kim ${ }^{2}(\mathbb{D})$ and SeungHwan $\mathrm{Yi}^{2, *(1)}$ \\ 1 Department of Control \& Instrumentation Engineering, Korea National University of \\ Transportation (KNUT), 50 Daehakro, Chungjushi, Chungbuk 27469, Korea; humaira27@ut.ac.kr \\ 2 Department of Mechanical Engineering, Korea National University of Transportation (KNUT), 50 Daehakro, \\ Chungjushi, Chungbuk 27469, Korea; wlsgh0614@naver.com \\ * Correspondence: isaac_yi@ut.ac.kr; Tel.: +82-43-841-5129
}

Received: 3 August 2018; Accepted: 29 August 2018; Published: 1 September 2018

\begin{abstract}
This paper discusses the output characteristics of the sensor response of infrared ethanol gas detectors based on incident radiation intensity. Sensors placed at each focal point of two elliptical waveguides were fabricated to yield two module combinations and to verify the output characteristics. A thin Parylene-C film was deposited onto the reflector surfaces of one module. The thermal properties were compared between the sensor (2.0 Ø) and sensor with a hollow disk (1.6 Ø), the disk being mounted at the end of one detector. The fabricated sensor modules were placed inside a gas chamber. The temperature was increased from $253 \mathrm{~K}$ to $333 \mathrm{~K}$, over the concentration range from 0 to $500 \mathrm{ppm}$. As the temperature increases by $10 \mathrm{~K}$, the output of sensor $(2.0 \varnothing)$ without and with Parylene-C coating typically increased by $70 \mathrm{mV}$ and $52 \mathrm{mV}$, respectively. However, the sensor output with the hollow disk showed an average decrement of $0.8 \mathrm{mV} / 50 \mathrm{ppm}$ and $1 \mathrm{mV} / 50 \mathrm{ppm}$ for module without and with Parylene-C deposition, respectively. For concentrations higher than $50 \mathrm{ppm}$, the estimation error was around $\pm 5 \%$. Further, the sensitivity to temperature variation and the absorbance of infrared (IR) reflection was found higher for Parylene-C coated module.
\end{abstract}

Keywords: ethanol gas; Non-Dispersive Infrared (NDIR); optical waveguide; dual-elliptical structure; temperature compensation

\section{Introduction}

Random breath testing and use of ignition interlock devices can reduce the risk of drunk driving and motor vehicle accidents by $20 \%$ [1] as the number of alcohol-impaired traffic fatalities has increased alarmingly [2]. Research has been conducted in several phases to observe human interaction with alcohol detection technology and also, the commercialization of alcohol detectors. The non-dispersive infrared (NDIR) gas detection method is advantageous over existing electrochemical sensors [3], metal oxide sensors $[4,5]$ in the aspects of superior gas specificity, simpler fabrication, selectivity and quicker response time. Furthermore, periodic calibration is not required for NDIR sensors, unlike other proposed systems [6]. Any gas molecule under the influence of infrared (IR) light of intensity equivalent to a permissible vibrational transition, absorbs the light at a specific wavelength and suffers a transition between the different vibrational energy levels. The absorption strength determines the concentration of the target gas in a gas cell or chamber [7,8]. Infrared detectors with narrow band-pass filters respond to a very specific infrared spectrum for a particular gas. Even though prevalently used for $\mathrm{CO}_{2}$ [9,10], $\mathrm{CH}_{4}$ [11-13] and other gas detection [14], a few ethanol detectors pertaining to the same method have been reported so far [15-18]. The response of such sensors is affected by ambient 
temperature [14] implying that some temperature compensation method must be included in the sensor system. While the selectivity depends on the specific wavelength range for IR absorption, both sensitivity and detection limit can be enhanced by increasing IR intensity and optical length [14,19]. To achieve chemical, mechanical and electrical robustness [20,21], Parylene-C is widely used as a thin film coating [22-24]. In addition to securing chemical resistance and preventing vapor condensation on the surface of the reflectors, the sensor with the thin film coating was reported to show higher absorbance and reduced estimated gas concentration error, being significantly sensitive to temperature variation [25].

This research was conducted with the aim to build a compact, robust and stable NDIR alcohol detector acknowledging both the advantages and limitations of such an application. A unique dual-elliptical ethanol detector is designed in this research with two identical IR detectors, except one detector has an external hollow disk mounted on them because the performance of a sensor is extensively affected by optical waveguide design and intensity of light. Each of the detectors were placed on the focus of each ellipse while the source at the common focus. Afterwards, two similar modules were constructed but the reflector surfaces of one module utilize the protective properties of thin film Parylene- $C$ coating and the results were justified. Then the temperature dependency was compensated, and finally this article verifies the measurement accuracy according to different light intensity incident on the two detectors.

\section{Theoretical Background}

\subsection{Basic Structure and Principle of Non-Dispersive Infrared Gas Sensor}

The basic structure of an NDIR gas sensor system has been illustrated in the articles [19,26] and it comprises the following components: a broadband IR source along with its driving circuit, an optical waveguide, and an IR detector with a narrow band-pass filter attached to it. The operation of an NDIR gas sensor lies in the principle that when broadband radiation is emitted by an IR source, the narrow band-pass optical filter turns the IR detector into a much specific narrow band detector that screens out all the radiation except for a particular wavelength, which is absorbed by the sample gas. Typical absorption spectrum of ethanol is at $3.45 \mu \mathrm{m}$ [27]. The IR absorption spectrum of ethanol and transmission spectrum of narrow band-pass filter which is used in this research, are plotted simultaneously in Figure 1. The overlapping of the two spectra significantly improves the detection of target gas at the mentioned peak wavelength.

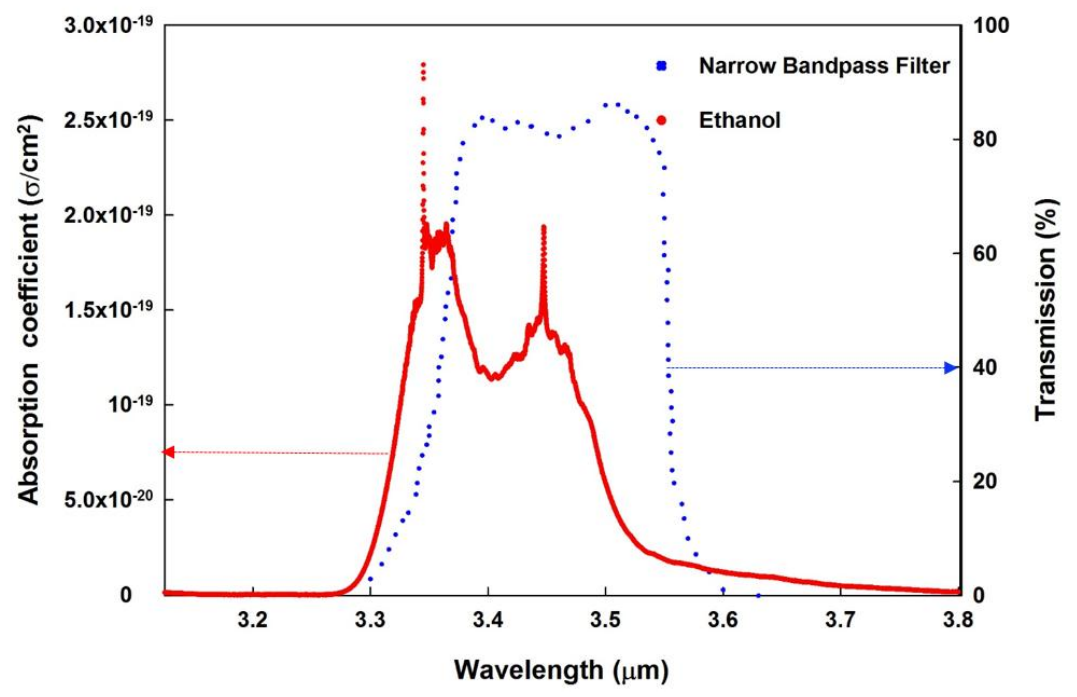

Figure 1. Superimposed transmission spectrum of the narrow band-pass filter on the absorption spectrum of ethanol gas. 
The absorption of water is five times less than that of ethanol at $3.45 \mu \mathrm{m}$ [28]. Also, the optical structure with Parylene-C coating has lower permeability to moisture and chemical resistance can be secured by preventing contamination and corrosion due to contact with air [21].

\subsubsection{Infrared Source}

Regarding the selection of a light source (IR source), the intensity at the absorption wavelength of the molecule and the bandwidth of the light source are very vital. Ethanol gas has the highest absorption rate for infrared rays of $3.45 \mu \mathrm{m}$. Although theoretically ideal, it is not feasible to use an IR source emitting at a particular wavelength. Instead, a wideband blackbody infrared emitter is chosen. In the case of continuous wave light source for IR emission, the filament is heated to a high temperature to emit light, which is termed as the blackbody radiation theory. When a black body is the emission source, the total energy transmitted $\mathrm{I}_{\mathrm{T}}$ across all wavelengths, is in accordance with the Stefan-Boltzmann law [29-31]:

$$
I_{T} \propto\left(T_{\text {body }}^{4}-T_{\mathrm{amb}}^{4}\right),
$$

where $T_{\text {body }}$ is the temperature of the black body, $T_{\mathrm{amb}}$ is the temperature of the surroundings.

Planck's radiation theory describes the emission energy density function [31]. While the temperature of a blackbody emitter increases, the overall radiated energy is enhanced. Further, the peak of the radiation curve shifts to shorter wavelengths as a consequence of the increase in temperature. While Planck's radiation theory yields the maximum emission of energy, the product of the peak wavelength and the temperature is constant [32].

The infrared light source (MILR17-900, developed by Intex, distributed by Importec, Polcenigo, Italy ) used in this research, continuously imitates the radiation pulses at a widely spectral range of 0 to $10 \mu \mathrm{m}$ and the emission spectrum resembles that of blackbody. Table 1 lists the features of the IR source.

Table 1. Features of infrared (IR) source (MILR17-900).

\begin{tabular}{cc}
\hline Parameter & Typical Value \\
\hline Spectral output range & $1-10 \mu \mathrm{m}$ \\
Emitter Surface Area & $1.7 \times 1.7 \mathrm{~mm}^{2}$ \\
Working temperature & $750{ }^{\circ} \mathrm{C}$ \\
Power consumption & $980 \mathrm{~mW}$ \\
Modulation frequency & $0-100 \mathrm{~Hz}($ optimized at $2.5 \mathrm{~Hz})$ \\
Warm up time & $<30 \mathrm{~ms}$ \\
Lifetime & $>5000 \mathrm{~h}$ at $750{ }^{\circ} \mathrm{C}$ \\
\hline
\end{tabular}

\subsubsection{Optical Waveguide}

The intensity of light transmitted through an absorbing medium can be obtained by the Lambert-Beer law given in Equation (2) [33]:

$$
I_{d}=I_{0} \exp (-\alpha x l), I_{\mathrm{d}}=I_{0} \exp (-\beta(T) x),
$$

where $\mathrm{I}_{0}$ is the initial incident light intensity, $\beta(T)$ is the product of the gas absorption coefficient $(\alpha)$ and the optical path length (1) and $x$ is the ethanol concentration (ppm).

This equation represents the co-dependence between optical irradiance and geometric parameters. The light intensity received at the detector can be determined by the gas concentration, optical path length and absorption coefficient of target gas, provided that the characteristics of the target gas have temperature dependent absorption properties. Hence, a larger optical length will enhance the sensitivity of the sensor at the same target gas concentration.

The characteristic absorption of a gas occurs in the analysis cell structure. Although larger optical path length is desired, the dimension of the chamber must be within acceptable range [34]. 
Several waveguide structure of various shapes and sizes have been studied over the years-cylindrical with circular cross section [26], spherical [9], combination of pairs of off-axis parabolic mirrors and right-angle mirrors [35] etc. Elliptical structures, in particular, can be characterized by the property that light traveling from one focus reaches the other focus. This shape was utilized for offering extended optical path length and multiple reflections of light from IR source to the detector end. A larger aperture combined within a smaller structure provides compactness [15,36]. Using dual wavelength differential principle, both symmetrical $[12,37]$ and asymmetrical [38] single ellipsoid was optimized to achieve high sensitivity of the methane detector. Two intersecting symmetrical ellipsoid structure, similar to the proposed one in this paper were reported in [11,16]; source and detector are placed in focus of separate ellipse in [11] whereas the source is placed in a common focus and each detector at the remaining two focuses in [16].

The ethanol sensor structure presented in this paper can be termed unique due to the construction differences. This structure utilizes two identical ethanol detectors, each placed at the non-intersecting focus of the respective ellipsoid. This arrangement is implemented with one detector being intact at the upper part of the structure while the other is partially blocked by a hollow disk at the lower part which results in difference in received irradiation energies at the detector end. Hence, even though both the sensors take part in ethanol detection, the unblocked and partially blocked structures can be used to demonstrate the output characteristics of the sensors in case of different incident energy intensity. Both ellipsoids share a common focus where the IR source is placed while the detectors are on the non-intersecting focus. Moreover, thin film of hydrophobic Parylene-C is coated on the detectors of one of the two modules developed, in order to analyze and verify the improvement of electrical response along with preventing the reflector surfaces from decaying.

This design principle is based on the characteristics of the output of the sensor with varying temperature and concentration. The radiation energy intensity is proportional to the change in ambient temperature, so as the temperature is changed the output characteristic also change. The output voltage can be expressed as an exponential equation derived from Lambert-Beer's law and are dependent on ethanol concentrations and temperature as given in Equation (3) [17]:

$$
\operatorname{VdF}(T, x)=\operatorname{VoF}(T)[\exp (-\beta(T) x)]
$$

where $\operatorname{VdF}(T, x)$ is the output voltage at a certain temperature and concentration of ethanol gas, $\operatorname{VoF}(T)$ is the output voltage at 0 ppm concentration.

In obtaining the parameters from regression analysis of voltage response curve using Equation (3), the concentration can be precisely estimated using the parameters in the following [16]:

$$
x_{\mathrm{ppm}}=-\frac{\ln \left(\frac{V d F}{V o F}\right)}{\beta(T)}=-\frac{\ln (a)}{\beta(T)},
$$

Here, $\ln (a)$ can be expanded in the form of a standard series and ethanol concentration can be estimated by substituting the series in Equation (4) [16]. Precise measurement and the trend of response of initial output voltage at $0 \mathrm{ppm}$ concentration, $\beta(T)$ and the output voltage curves after injection of ethanol at different ppm levels over a varying temperature range will determine the accuracy of measurement.

\subsubsection{Infrared Detector}

The energy transmitted by an IR source is received at the IR detector. The detector used in our analysis is a thermopile detector. When the thermopile detector is integrated with an appropriate band-pass filter, it can measure certain gases such as $\mathrm{CO}_{2}, \mathrm{CO}, \mathrm{CH}_{4}$, ethanol etc. The amount of light received by the detector is converted to the output voltage depending on the temperature difference 
between the high and low temperature contacts. The output voltage of the thermopile detector is governed by Equation (5) [39]:

$$
V=\int_{T_{\text {Low }}}^{T_{\text {High }}} \Delta \alpha_{S} \mathrm{~d} T
$$

where $T_{\mathrm{High}}$ is the temperature of the high-temperature contact, $T_{\text {Low }}$ is the temperature of the low-temperature contact, and $\alpha_{S}$ is the Seebeck constant.

The HIS A21E3.45G5600s manufactured by HEIMANN Sensors ${ }^{\odot} \mathrm{GmbH}$ (Dresden, Germany) used in this experimental study can operate at a temperature range of -40 to $120^{\circ} \mathrm{C}$ and offers an integrated linear temperature sensor output of $15 \mathrm{mV} /{ }^{\circ} \mathrm{C}$ sensitivity. The detector is attached with narrow band-pass filter centered at $3.45 \mu \mathrm{m}$ for precise ethanol gas detection. To overcome noise interference from external amplification circuit in lower gas concentration, thermopile detectors are often integrated with Application Specific Integrated Circuit (ASIC) chip. This reduces noise component and enhances the output properties of thermopile.

\subsubsection{Geometric Realization of an Elliptical Cell}

Each of the ellipsoid structure is a symmetrical and identical. The semi-major and semi-minor axis of the geometrical shape can be denoted by a and $b$. The IR source is placed at one focal point at a distance $c=\sqrt{a^{2}-b^{2}}$ and the detector is placed at the other focal point; at a distance $2 c$ from the source, on the major axis. The surface area of the detector area is $S$ and $K$ is the reflectivity of the structure [37]. The radiant intensity from the source incident at a certain angle $I(\theta)$ and radiant flux received at the detector $\phi$ end can be described by the following equations [37]:

$$
\begin{gathered}
I(\theta)=I_{a} \cos \theta \\
\phi=I_{a} \frac{S}{(2 c)^{2}} \mathrm{e}^{-\alpha(2 c)}+2 \pi I_{a} K \int_{\theta_{1}}^{\theta_{2}} \mathrm{e}^{-\alpha l} \cos ^{2} \theta \mathrm{d} \cos \theta
\end{gathered}
$$

where $I_{a}$ is the radiant along the axes.

According to Equation (7), the first part in the equation corresponds to flux received without any reflection while the second part corresponds to the flux received after reflection [38]. From this equation, it can be assumed that if the detector area is blocked by any external means, received flux is altered.

\section{Sensor Module Fabrication and Analysis}

With the aim of finding the optimum combination to compensate for the effect of different energy densities and sensitivity to ambient temperature variation, two sensor modules were constructed. A thin film of hydrophobic Parylene-C was deposited on the reflectors of one of the sensor modules, as a preventive measure for water condensation and chemical corrosion. To verify the output characteristics of the infrared detector according to the amount of incident light, a hollow disk was mounted in front of one of the two infrared detectors present in the waveguide structure-having a constant optical path length in both the sensor modules. One detector without any hollow disk $(2.0 \varnothing)$ is placed in the upper part, while another equipped a hollow disk of $6 \mathrm{~cm}(1.6 \varnothing)$ was placed in the lower part of the sensor structure [17]. The hollow disk was mounted to examine the amount of incident light on the infrared detector according to IR intensity. Upon completion, the module was used in the experiment to study the characteristics of the ethanol gas sensor at various temperatures and concentrations.

\subsection{Sensor Module Design Considerations and 3D Modelling}

Even though the proposed waveguide has two identical symmetrical ellipsoids, the separation between the major axis affects the light reaching the detector. The modeling and optimization of the cell shape has already been analyzed in [40]. The optimum separation angle between the two major axis is found to be 30 degree. This angle was chosen as a tradeoff between maximum irradiance 
and incident ray count. Maximum irradiance also obeys the condition that the IR source is placed at the focal point. Ray-tracing simulations showed multiple reflections along the path length which ensures larger output voltage and improved sensitivity. The length of the major and minor axis of each ellipse is $154.95 \mathrm{~mm}$ and $20 \mathrm{~mm}$. During the initial simulation, the light is transmitted with a source power $600 \mathrm{~mW}$, the number of incident rays were 100,000 and the reflectivity of the elliptical surface is 95\%. The 3-D modeling of the fabricated sensor module is exhibited in Figure 2. The IR source was placed on the common focus of the dual elliptical waveguide while the detectors with no disk (2.0 Ø) and with disk $(1.6 \varnothing)$ were modeled to be on the remaining focal point of each ellipse.

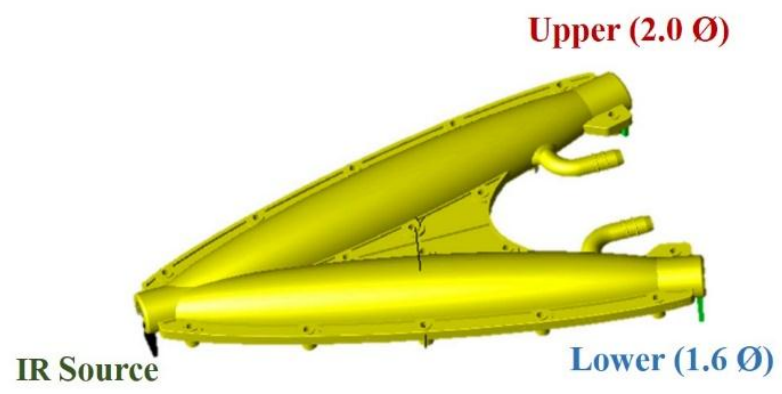

Figure 2. 3-D modeling of the sensor module.

The 3-D shape was designed using Solid-works ${ }^{\circledR}$ (Dassault Systèmes, Vélizy-Villacoublay, France) and the simulation was performed using Trace-Pro ${ }^{\circledR}$ (Lambda Research Corporation, Littleton, CO, USA). The model was later fabricated in a highly precise molding structure to build the optical waveguide with functional plastic. The fabricated dual elliptical structure with the source and detectors assembled onto it is mounted on a printed circuit board shown in Figure 3a,b. The circuitry was placed separately from the waveguide in order to keep the signal to noise ratio unaffected.

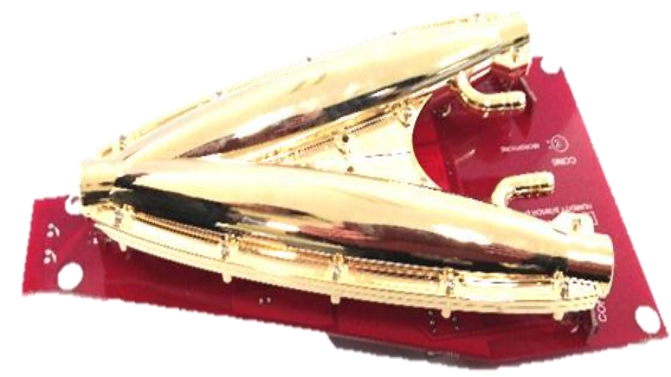

(a)

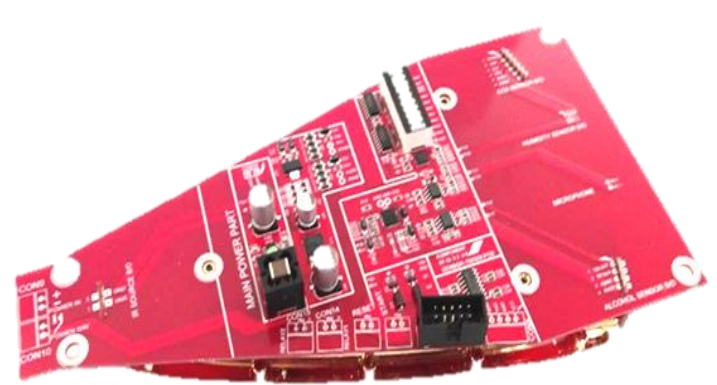

(b)

Figure 3. (a) Fabricated dual elliptical ethanol sensor module; (b) Printed circuit board below the dual elliptical structure.

\subsection{Signal Conditioning Circuit}

For signal conditioning, the two electrical power supply-analog and digital are kept apart to acquire the stability. This is because the IR source is modulated at a high input power of $600 \mathrm{~mW}$. A variable resistor is used to adjust the peak analog supply voltage of $9 \mathrm{~V}$ applied to the IR source. The on- and off-time pulses are controlled by the MicroController Unit (MCU). The integrated IR detectors with ASIC chip were installed. A pre-amplifier is housed in the same packaging. The detector signals are enlarged by a pre-amplifier (MAX4239, Maxim Integrated Products Inc., San Jose, CA, USA) which offers low drift and ultra-low offset. The analog signals are converted into digital ones by an A/D converter after the signals have passed through two stages of differential OP-AMP. This amplifier helps to measure the difference between the output voltages more precisely and improving the sensitivity. Output signals are transmitted through the RS-485 (Analog Devices, Inc., Norwood, 
MA, United States) chip to the computer for further analysis. The block diagram of the circuitry is presented in Figure 4. The analog signals are represented as dotted lines whereas solid lines imply digital signals.

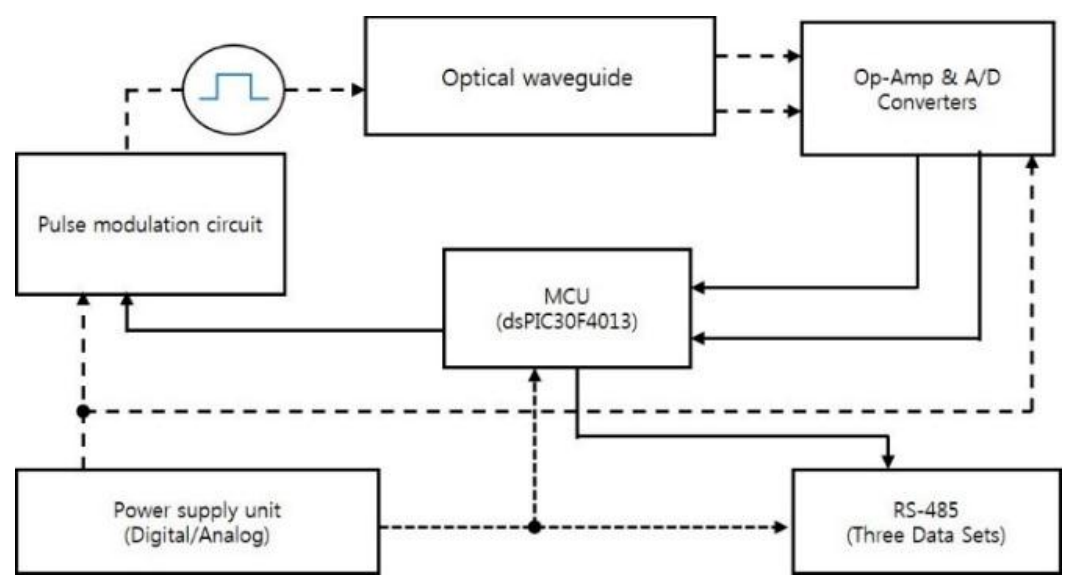

Figure 4. Block diagram of signal conditioning circuit.

\subsection{Simulation Result}

The infrared active region of $1.2 \mathrm{~mm} \times 1.2 \mathrm{~mm}$ was formed at the center of the infrared sensor shown in Figure 5, and the simulation result was obtained assuming that the active region absorbs $100 \%$ of the incident light. Reflecting on the initial conditions, the simulated results of the incident energy absorbed by different waveguide structures are shown in Figure 5a,b. In Figure 5b, the amount of incident light decreases as the size of the hole in the disk decreases. This phenomenon can be explained by Equation (7). When the hollow disk is mounted before the detector, the angular range of incident light emission $\theta$ becomes limited by the change in the area of incidence. Consequently, the received energy at the detector will be less than that of intact detectors. In the case of an infrared detector $(2.0 \varnothing)$ without a hollow disk, the radiant power per unit area at the detector was observed to be $0.030 \mathrm{~W}$, whereas for a detector with a hollow disk $(1.6 \varnothing)$ the power simulated was $0.026 \mathrm{~W}$.

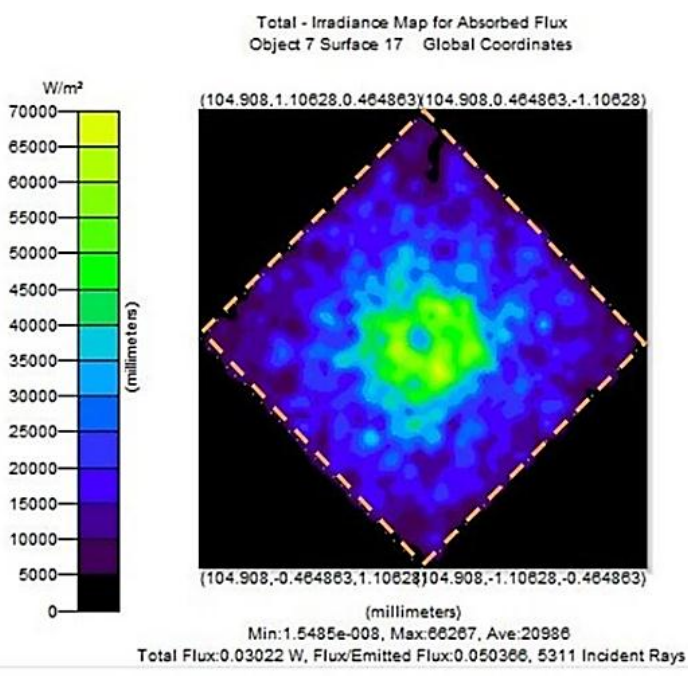

(a)

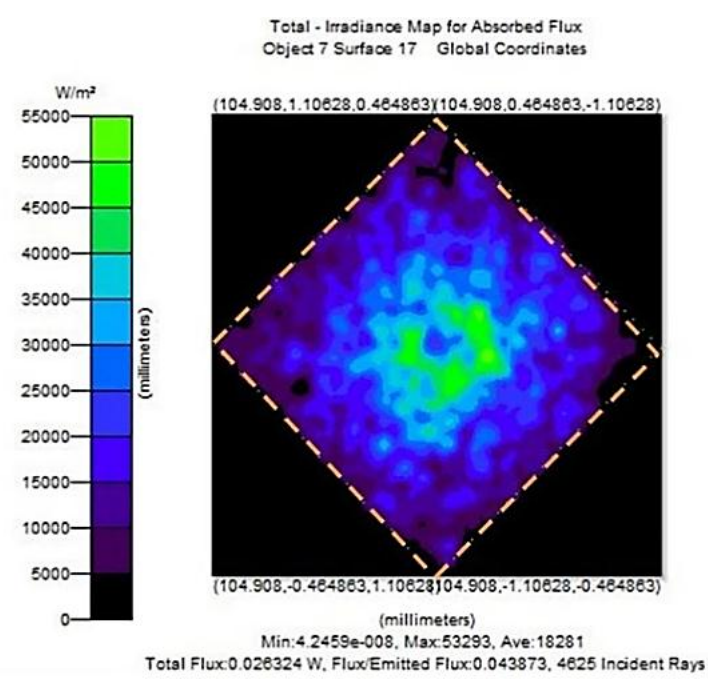

(b)

Figure 5. Initial simulation results (a) normal structure (2.0 Ø); (b) with hollow disk (1.6 Ø). 


\section{Experimental Method}

To evaluate the output characteristics of the sensor module according to the temperature and the ethanol gas concentration, a measurement system as shown in Figure 6 was set up. First, the sensor module was inserted into a closed gas chamber inside the thermo-hygrostat, and the temperature was adjusted from $253 \mathrm{~K}$ to $333 \mathrm{~K}$ at step intervals of $10 \mathrm{~K}$ except at the temperature at $298 \mathrm{~K}$. After adjusting the temperature, more than $4 \mathrm{~h}$ had passed before the thermal equilibrium state of the sensor module was attained. When the variation of the output of the temperature sensor was within $\pm 5 \mathrm{mV}$ of the previous experimental result, the mass flow controller (MFC) of gas was turned on. Subsequently, the gas inside the chamber was purged with high-purity nitrogen $(99.9999 \%$, class 6) to reach $0 \mathrm{ppm}$ by waiting for $1 \mathrm{~h}$ or more. The experiments were then carried out by feeding a high concentration of ethanol gas through the mass flow controller of gas into the sealed gas chamber, from 0 to $500 \mathrm{ppm}$ in 50 ppm step units.

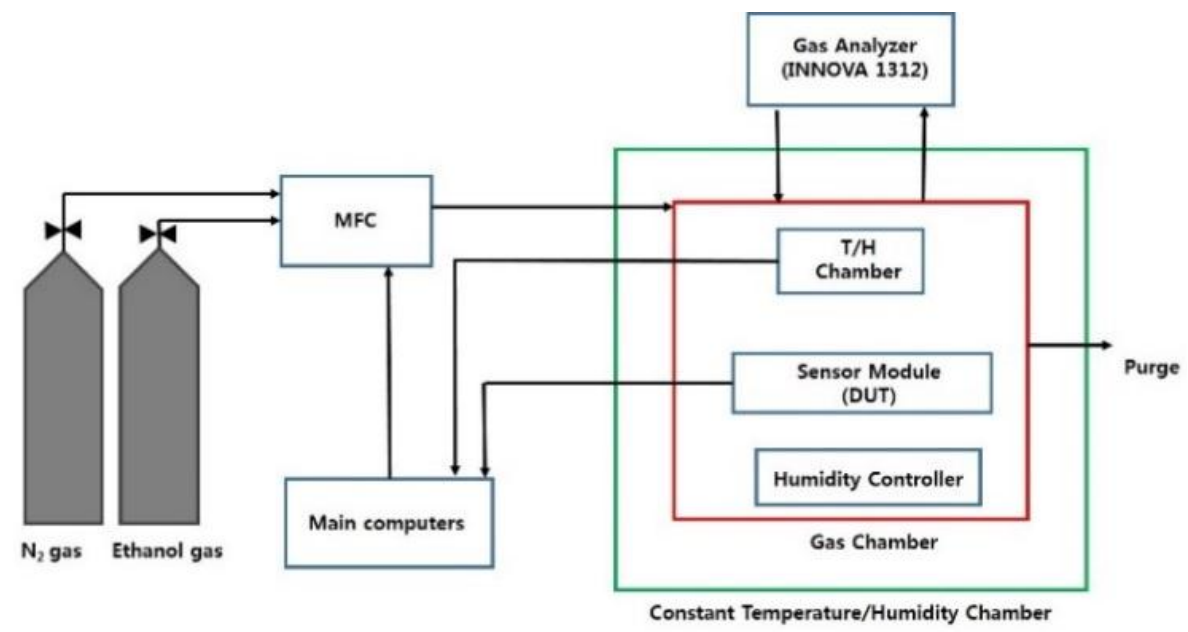

Figure 6. Block diagram of experimental setup. MFC: Mass Flow Controller; DUT: Device Under Test.

A multi-gas analyzer (INNOVA-1312, LumaSense Technologies A/S, Ballerup, Denmark) was used to standardize the successive measurements of ethanol concentration with a precision of $1 \mathrm{ppm}$ or less. The gas analyzer is calibrated and attached with special filters for accurate ethanol measurement. The connection from the analyzer to the gas chamber is properly insulated. The output of the sensor module according to the concentration was recorded as a voltage in the computer, and the corresponding experimental data was saved using the RS- 485 communication.

\section{Result \& Discussion}

\subsection{On-Off and Response Time}

The IR source is modulated for pulse generation and recorded on-off time is shown in Figure 7a. The optimum on-off time of IR source is found to be $100 \mathrm{~ms}$ and $300 \mathrm{~ms}$, respectively. The values were chosen after iterative experiments. It takes a while for the source to transmit IR energy to the detector, so that the sensor response can reach to the peak amplitude level but even if the on-time is further extended, the peak voltage does not increase significantly. On the other hand, if the off time is shorter, peak value of voltage cannot be reached. In case of longer off time, the data sampling duration will be higher. The response time of the ethanol sensor is observed to be $48 \mathrm{~ms}$ as seen in Figure $7 \mathrm{~b}$. 


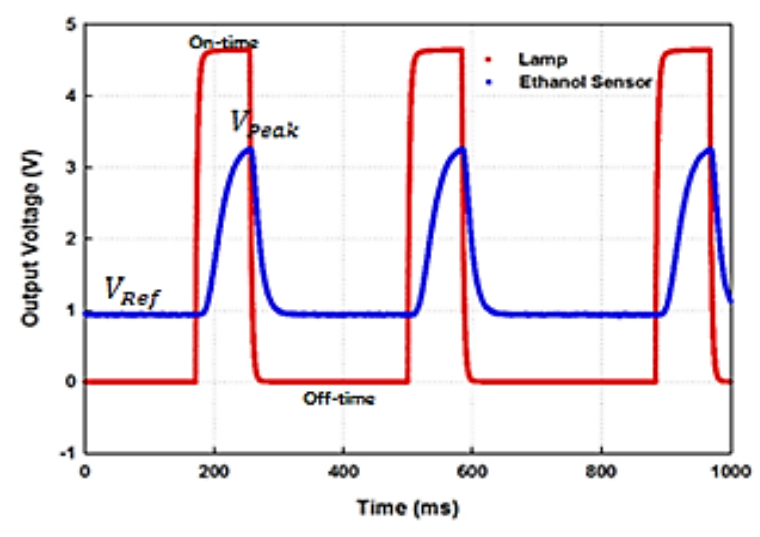

(a)

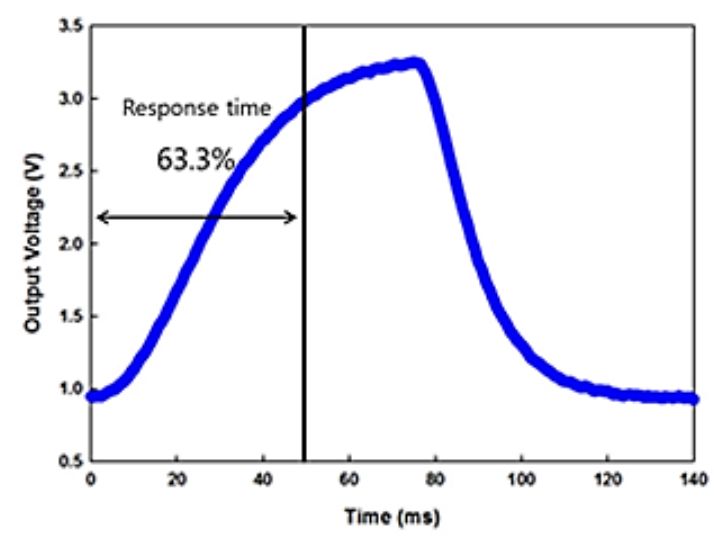

(b)

Figure 7. (a) On-off time of the IR source and response of the sensor; (b) response time of the sensor.

\subsection{Output in Absence of Target Gas}

When the gas chamber was purged with $\mathrm{N}_{2}$ gas to obtain $0 \mathrm{ppm}$ concentration of target gas, the output voltages were also recorded and downloaded. The signal values at different temperature for each detector of each module was plotted in Figure 8. It is evident that even in the absence of target gas the outputs are different. The initial output voltage varies because of the constructional differences between the sensor modules. These differences among the results can again be explained by Equation (7). As the temperature rises from $253 \mathrm{~K}$ to $333 \mathrm{~K}$ at $10 \mathrm{~K}$ interval (including the interval between $273 \mathrm{~K}$ and $298 \mathrm{~K})$, the initial output voltage of the $(2.0 \varnothing)$ sensor with no Parylene-C increases by $70 \mathrm{mV}$ on average. With the hollow disk being mounted (1.6 Ø), the same sensor module output rises by $33 \mathrm{mV}$, as a result of change in light transmitting angle with respect to the major axis of elliptical waveguide reaching the detector end. The Parylene- $C$ deposited sensor module with structure $(2.0 \varnothing)$ increases by $52 \mathrm{mV}$ with each ascending temperature setting. However, at the output of the detector with a hollow disk, we observed that the initial output voltage increases by $24 \mathrm{mV}$ on average with the increase in temperature. The increment in voltage showed a temperature dependency, resulting in a coefficient of determination, $R^{2}=0.9833$. The linearity quantifies the output variation with respect to IR source power. Conversely, the prototype with Parylene-C deposition showed linear temperature-dependent characteristics with a higher coefficient of determination, $R^{2}=0.9975$ along with higher output voltage. The output voltages at $0 \mathrm{ppm}$ for each module are presented in Figure $8 \mathrm{a}, \mathrm{b}$.

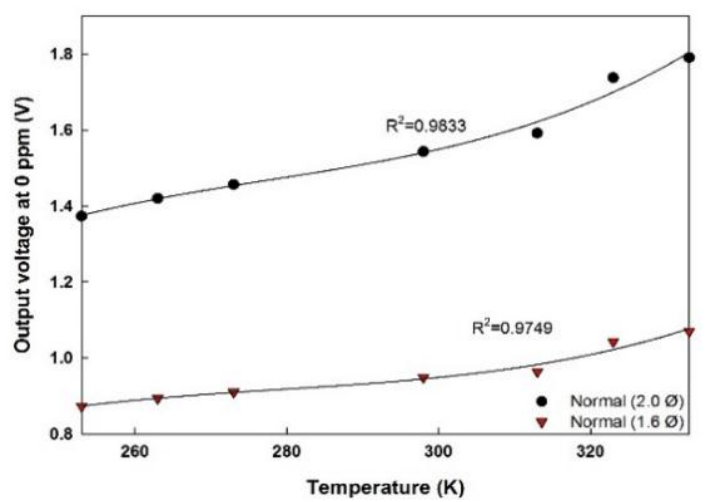

(a)

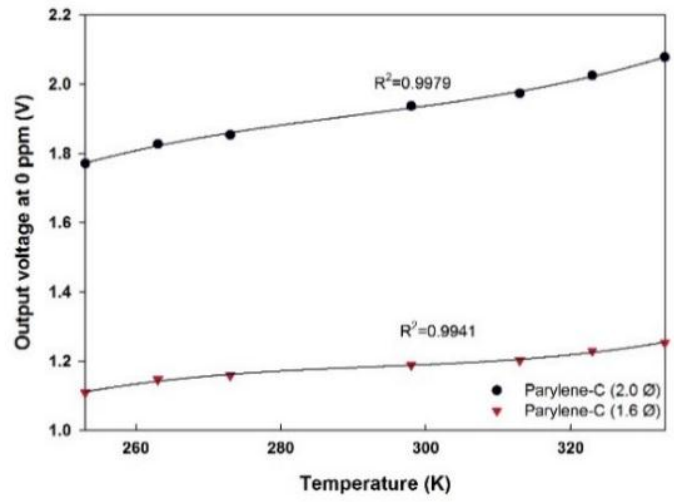

(b)

Figure 8. Output voltages at $0 \mathrm{ppm}$ ethanol concentration with varying temperature obtained from (a) normal sensor module; (b) sensor module with Parylene-C deposition. 
The output voltages increase as the ambient temperature increases from $253 \mathrm{~K}$. This can be characterized by Stefan-Boltzmann law in Equation (1). As the ambient temperature rises higher, the temperature of the source also increases resulting in a greater difference between the source and ambient temperature. Consequently, according to Equation (1), transmitted light intensity improves proportionally to the difference between the fourth powers of the two temperature. The output voltage at the detector end is also a measure of incident light intensity. Hence the output voltages enhance with the increase in ambient temperature conditions.

The $\beta(T)$, the product of the gas absorption coefficient and optical path length can be determined by the regression analysis of the second-order polynomial function of measured temperature. The values of the initial voltage and gas absorption coefficient recorded at each temperature for both modules are given in Table 2.

Table 2. Values of initial voltage and gas absorption coefficients at various temperatures.

\begin{tabular}{ccccc}
\hline Temp. (K) & \multicolumn{2}{c}{ Normal (2.00 Ø) } & \multicolumn{2}{c}{ Parylene-C (2.00 Ø) } \\
\hline & $\mathbf{V}_{\mathbf{~ o F}} \mathbf{( T )}$ & $\boldsymbol{\beta}(\mathbf{T})$ & $\mathbf{V}_{\mathbf{~ o F}} \mathbf{( T )}$ & $\boldsymbol{\beta}(\mathbf{T})$ \\
\hline 253 & 1.3736 & $5.9 \times 10^{-5}$ & 1.7712 & $5.0 \times 10^{-5}$ \\
263 & 1.4204 & $5.6 \times 10^{-5}$ & 1.8271 & $5.1 \times 10^{-5}$ \\
273 & 1.4572 & $5.1 \times 10^{-5}$ & 1.8536 & $4.8 \times 10^{-5}$ \\
298 & 1.5435 & $5.4 \times 10^{-5}$ & 1.9375 & $5.0 \times 10^{-5}$ \\
313 & 1.5920 & $5.3 \times 10^{-5}$ & 1.9731 & $4.8 \times 10^{-5}$ \\
323 & 1.7378 & $5.1 \times 10^{-5}$ & 2.0255 & $4.8 \times 10^{-5}$ \\
333 & 1.7909 & $5.3 \times 10^{-5}$ & 2.0784 & $5.1 \times 10^{-5}$ \\
\hline
\end{tabular}

Table 2 shows that the product of absorption coefficient and optical path length doesn't show much temperature dependency. However, the values of $\beta(T)$ are stored and are formulated as a second order polynomial function of the temperature setting.

\subsection{Temperature Dependency}

The output voltages received over the range of concentration used in this experiment for each temperature setting are stored and plotted. The signal curves follow temperature fitting according to Equation (5) depending on the concentration value. When the ethanol gas is injected inside the chamber, the absorption of the target gas in the optical waveguide under the influence of IR light is initiated around $3.45 \mu \mathrm{m}$. Due to the exponential relationship between the output voltage and concentration corresponding to Equation (5), the sensor output decreases as the amount of ethanol is increased. Also, as described in the previous section, the increase in ambient temperature yields in the improved radiation energy intensity. The output voltages increase at higher temperature for the same ethanol concentration injected in the gas chamber but decay with increased gas absorption at the measured temperature.

The experimental results show that, with a hollow disk attached, when the concentration is higher than $10 \mathrm{ppm}$, the output of the sensor without thin film decreases by $0.8 \mathrm{mV} / 50 \mathrm{ppm}$ interval on average, over the range of ethanol concentration tested in this experiment. However, the output voltage of the sensor module with deposition decreases by $1 \mathrm{mV} / 50 \mathrm{ppm}$ interval on average for concentrations above $10 \mathrm{ppm}$. Compared to the module with no thin film deposition, Parylene-C module shows improved voltage response and higher sensitivity to varying concentration and also to temperature change since it can minimize the effect of infrared scattering caused by adhesion of water vapor. The output voltages of Parylene-C deposited module are presented in Figure 9a,b. 


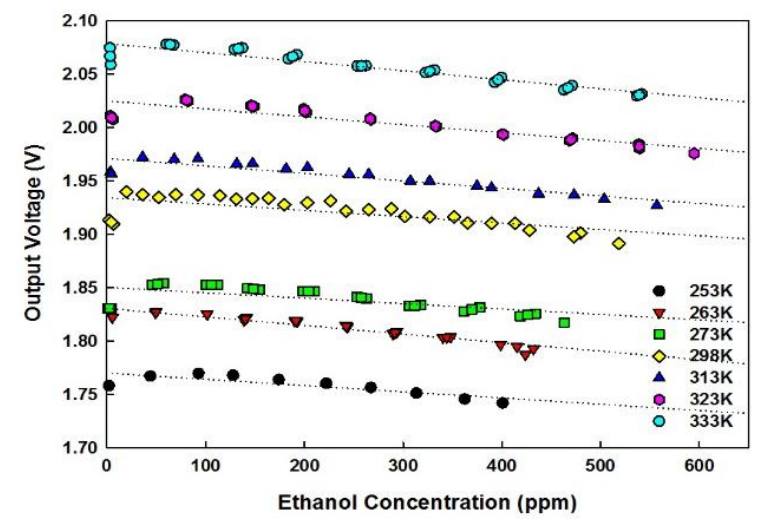

(a)

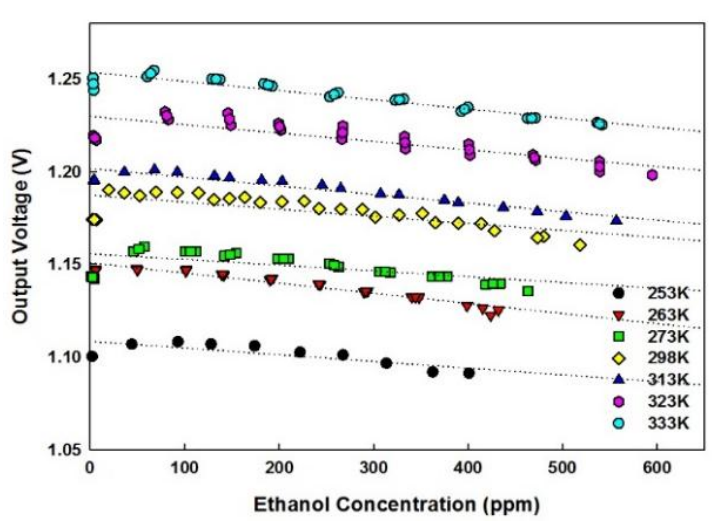

(b)

Figure 9. Parylene-C sensor output voltages of (a) detector with no disk (2.0 Ø); (b) detector with hollow disk (1.6 Ø).

Therefore, along with the voltage contribution from IR source which is independent of the ethanol gas absorption but depends on ambient temperature, system response is also affected by absorption of ethanol around $3.45 \mu \mathrm{m}$ with respect to varying temperature and concentration.

\subsection{Temperature Compensation}

The ethanol concentration can be evaluated by using the expanded Equation (4). The initial output voltages at $0 \mathrm{ppm}$ concentration show a high dependency on temperature. The average voltage ratios calculated at the measured temperature is constant over the entire concentration range. The ratios are implemented using 3rd order polynomial equations with varying ambient temperature. Again, since the denominator of the Equation (4) is almost constant at a certain temperature in the range in which the experiments are conducted, it is programmed in the MCU as a second order polynomial of ambient temperature. The estimated concentration is solely temperature reliant and can be measured accurately. The temperature compensation algorithm was formulated [10] and ethanol concentration was estimated at each measured temperature. Despite the temperature compensation, an offset from the temperature-compensated concentration occurs from the real concentration due to the energy received by the band-pass filter at wavelengths other than at $3.45 \mu \mathrm{m}$. Since the received thermal energy of an IR detector is directly proportional to the output voltage, the response is always observed with an offset, which depends on the thermal properties but is independent of the variation in the ethanol concentration. The difference between the actual concentration and the estimated concentration was obtained in the entire concentration range, and the average of the difference values was used to derive the correction value for every temperature point. These values were adjusted in the $\mathrm{MCU}$ algorithm to obtain accurate ethanol concentration. The corrected concentration is plotted in Figure 10a,b.

As evident from Figure 10, the concentration measured at both detector ends are almost similar. The corrected concentration shows reliable linearity corresponding to actual concentration in the gas cylinder which asserts that the temperature compensation algorithm has been successfully implemented. Although the received light intensity and subsequent output voltages of one detector are different from another, the ratio of the output voltages $\left(\frac{V d F}{V o F}\right)$ at the measured temperature is analogous for the entire concentration range. Therefore, precise estimation of ethanol concentration can be achieved irrespective of the IR intensity received by the detector. The consistent ratio of output voltages across the range of concentration ensures the stability of the sensor module. 


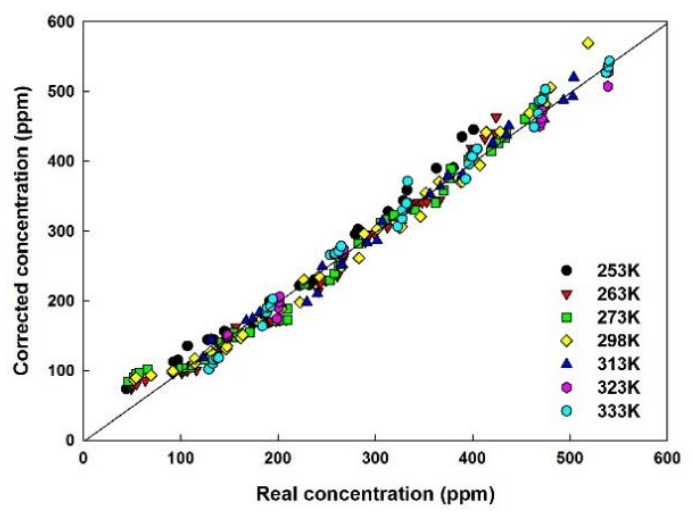

(a)

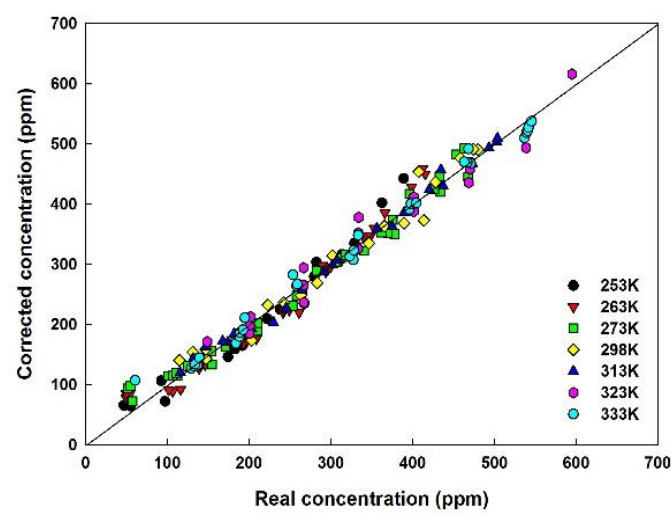

(b)

Figure 10. Corrected concentration of Parylene-C module at (a) detector end with no disk (2.0 Ø); (b) detector end with hollow disk (1.6 Ø).

\subsection{Error and Detection Limit}

The experiment was conducted for several months and the error in concentration was recorded. The error percentage for $50 \mathrm{ppm}$ concentration was much higher i.e., an error range starting from $10 \%$ or greater for both the sensors with and without deposition at a temperature less than or equal to $298 \mathrm{~K}$ was achieved.

Differences between initial calculations and regression analysis yielded higher error below $50 \mathrm{ppm}$ concentration. However, in the case of more than $50 \mathrm{ppm}$, the average error range is around $\pm 5 \%$. Although the error percentage is quite similar between the sensor with deposition and without deposition, since the sensor module deposited with Parylene-C has a larger output voltage change depending on the gas concentration than the normal sensor module, a more accurate estimated concentration can be obtained. The average error between each combination of the detector at $298 \mathrm{~K}$ for Parylene-C deposited module are plotted in Figure 11.

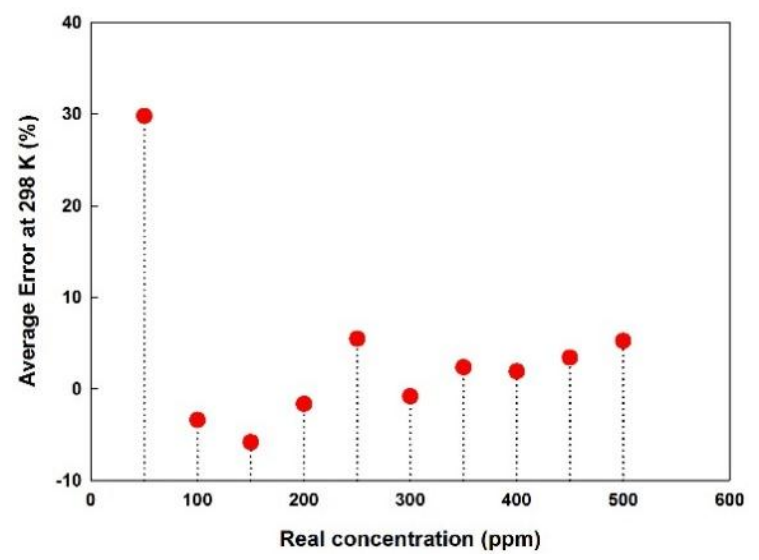

Figure 11. Average error of concentration measurement of Parylene-C module at $298 \mathrm{~K}$.

\section{Comparison and Conclusions}

The ethanol detector has been modeled and monitored for over two years and the progress is summarized in Table 3. While modeling using a reference detector sensor module of a higher accuracy can be achieved over entire concentration range, subsequent research work emphasized on verifying the sensor's response according to different incident ray intensities using two identical detectors at $3.45 \mu \mathrm{m}$. This has been established by partially blocking the detector i.e., incoming light intensity. Temperature compensation algorithm was implemented. The higher sensitivity and higher 
absorbance were obtained by using the properties of Parylene- $C$ coating. However, the geometrical shape of the waveguide structure is the same for all the mentioned works in Table 3. In this paper, the temperature characteristics, temperature compensation, and voltage output correction method were described according to the amount of incident light on an infrared sensor. The analysis from simulation allowed us to predict the amount of light entering the infrared sensor located at each focal point, and the output voltage was verified through experiments. The temperature dependent parameters were obtained to estimate ethanol concentration accurately irrespective of received IR light. It was confirmed that the mean error between the actual gas concentration and measured concentration after temperature compensation and calibration was typically $\pm 5 \%$, suggesting the applicability as a sensor module for alcohol detection.

Table 3. Comparison of design principle and performances among this sensor and previously reported ethanol sensors $[16,17,41]$.

\begin{tabular}{lll}
\hline Design Principle & Error (\%) & \\
\hline One ethanol and one reference detector & $\pm 5 \%$ & [16] \\
\hline One ellipsoid partially covered with tape & $\begin{array}{l}20-25 \% \text { at }<200 \mathrm{ppm} \\
-10 \text { to } 1 \% \text { at }>200 \mathrm{ppm}\end{array}$ & [41] \\
\hline Hollow disk (1.6 Ø) inserted before one detector & $\begin{array}{l} \pm 15 \% \text { at }<100 \mathrm{ppm} \\
-1.2-3 \% \text { at }>100 \mathrm{ppm}\end{array}$ & [17] \\
\hline $\begin{array}{l}\text { Hollow disk }(1.6 \varnothing) \text { inserted before one sensor, } \\
\text { detectors of one of the modules coated with }\end{array}$ & $\begin{array}{l}\text { More than } 10 \% \text { for } \leq 50 \mathrm{ppm} \\
\pm 5 \% \text { at }>50 \mathrm{ppm}\end{array}$ & This \\
\hline
\end{tabular}

Author Contributions: S.H.Y. conceptualized and supervised the research. J.H.K. conducted the experiment and summarized the results. H.H. prepared the draft and was involved in discussion of the results of this experiment.

Funding: This research was supported by the R\&D Center for Green Patrol Technologies through the R\&D for Global Top Environmental Technologies funded by the Ministry of Environment, Korea (MOE).

Conflicts of Interest: The authors declare no conflict of interest.

\section{References}

1. Elder, R.W.; Shults, R.A.; Sleet, D.A.; Nichols, J.L.; Zaza, S.; Thompson, R.S. Effectiveness of Sobriety Checkpoints for Reducing Alcohol-Involved Crashes. Traffic Inj. Prev. 2002, 3, 266-274. [CrossRef]

2. Blomberg, R.D.; Peck, R.C.; Moskowitz, H.; Burns, M.; Fiorentino, D. The Long Beach/Fort Lauderdale Relative Risk Study. J. Saf. Res. 2009, 40, 285-292. [CrossRef] [PubMed]

3. Shan, C.; Yang, H.; Han, D.; Zhang, Q.; Ivaska, A.; Niu, L. Electrochemical Determination of NADH and Ethanol Based on Ionic Liquid-Functionalized Graphene. Biosens. Bioelectron. 2010, 25, 1504-1508. [CrossRef] [PubMed]

4. Inyawilert, K.; Wisitsoraat, A.; Sriprachaubwong, C.; Tuantranont, A.; Phanichphant, S.; Liewhiran, C. Rapid Ethanol Sensor Based on Electrolytically-Exfoliated Graphene-Loaded Flame-Made In-Doped $\mathrm{SnO}_{2}$ Composite Film. Sens. Actuators B Chem. 2015, 209, 40-55. [CrossRef]

5. Sudha, M.; Radha, S.; Kirubaveni, S.; Kiruthika, R.; Govindaraj, R.; Santhosh, N. Experimental Study on Structural, Optoelectronic and Room Temperature Sensing Performance of Nickel Doped ZnO Based Ethanol Sensors. Solid State Sci. 2018, 78, 30-39. [CrossRef]

6. Modjtahedi, A.; Amirfazli, A.; Farhad, S. Low Catalyst Loaded Ethanol Gas Fuel Cell Sensor. Sens. Actuators $B$ Chem. 2016, 234, 70-79. [CrossRef]

7. Svanberg, S. Atomic and Molecular Spectroscopy: Basic Aspects and Practical Applications; Springer: Berlin, Germany, 2012; pp. 1-186.

8. Hummelgard, C.; Bryntse, I.; Bryzgalov, M.; Henning, J.A.; Martin, H.; Norén, M.; Rödjegard, H. Low-Cost NDIR Based Sensor Platform for Sub-Ppm Gas Detection. Urban Clim. 2015, 14, 342-350. [CrossRef] 
9. Hodgkinson, J.; Smith, R.; Ho, W.O.; Saffell, J.R.; Tatam, R.P. Non-Dispersive Infra-Red (NDIR) Measurement of Carbon Dioxide at $4.2 \mu \mathrm{m}$ in a Compact and Optically Efficient Sensor. Sens. Actuators $B$ Chem. 2013, 186, 580-588. [CrossRef]

10. Yi, S. Temperature Compensation Methods of Nondispersive Infrared $\mathrm{CO}_{2}$ Gas Sensor with Dual Ellipsoidal Optical Waveguide. Sens. Mater. 2017, 29, 243-252.

11. Tan, Q.; Pei, X.; Zhu, S.; Sun, D.; Liu, J.; Xue, C.; Liang, T.; Zhang, W.; Xiong, J. Development of an Optical Gas Leak Sensor for Detecting Ethylene, Dimethyl Ether and Methane. Sensors 2013, 13, 4157-4169. [CrossRef] [PubMed]

12. Xing, X.; Lei, Y.; Shang, W.; Du, L.; Guo, P. Theoretical Research on Ellipsoidal Structure Methane Gas Detection Based on Near Infrared Light Sources of PbSe Quantum Dots. J. Nano 2017, 2017, 1-7. [CrossRef]

13. Park, J.; Yi, S. Nondispersive Infrared Ray $\mathrm{CH}_{4}$ Gas Sensor Using Focused Infrared Beam Structures. Sens. Mater. 2011, 23, 147-158.

14. Sklorz, A.; Janßen, S.; Lang, W. Detection Limit Improvement for NDIR Ethylene Gas Detectors Using Passive Approaches. Sens. Actuators B Chem. 2012, 175, 246-254. [CrossRef]

15. Hök, B.; Pettersson, H.; Andersson, A.K.; Haasl, S.; Åkerlund, P. Breath Analyzer for Alcolocks and Screening Devices. IEEE Sens. J. 2010, 10, 10-15. [CrossRef]

16. Kim, J.; Lee, K.; Yi, S. NDIR Ethanol Gas Sensor with Two Elliptical Optical Structures. In Proceedings of the Eurosensors 2016, Budapest, Hungary, 4-7 September 2016; Volume 168, pp. 359-362.

17. Kim, J.; Shin, S.; Yi, S. Effects of Infrared Energy on Dual Elliptical NDIR Ethanol Gas Sensors. In Proceedings of the Eurosensors 2017, Paris, France, 3-6 September 2017; Volume 1, pp. 1-4.

18. Fonseca, L.; Rubio, R.; Santander, J.; Calaza, C.; Sabaté, N.; Ivanov, P.; Figueras, E.; Gràcia, I.; Cané, C.; Udina, S.; et al. Qualitative and Quantitative Substance Discrimination Using a CMOS Compatible Non-Specific NDIR Microarray. Sens. Actuators B Chem. 2009, 141, 396-403. [CrossRef]

19. Dinh, T.V.; Choi, I.Y.; Son, Y.S.; Kim, J.C. A Review on Non-Dispersive Infrared Gas Sensors: Improvement of Sensor Detection Limit and Interference Correction. Sens. Actuators B Chem. 2016, 231, 529-538. [CrossRef]

20. Kahouli, A.; Sylvestre, A.; Ortega, L.; Jomni, F.; Yangui, B.; Kahouli, A.; Sylvestre, A.; Ortega, L.; Jomni, F.; Yangui, B.; et al. Structural and Dielectric Study of Parylene C Thin Films. Appl. Phys. Lett. 2013, 152901, 1-3. [CrossRef]

21. Parylene Properties \& Characteristics. Available online: http://www.vp-scientific.com/parylene_properties. html (accessed on 4 June 2018).

22. Schubert, J.; Krabbe, A.; Birk, M.; Wagner, G. Parylene Anti-Reflection Coating of a Quasi-Optical Hot-Electron- Bolometric Mixer at Terahertz Frequencies. Infrared Phys. Technol. 2001, 42, 41-47.

23. Yasuda, H.; Chun, B.H.; Cho, D.L.; Lin, T.J.; Yang, D.J.; Antonelli, J.A. Interface-Engineered Parylene C Coating for Corrosion Protection of Cold-Rolled Steel. Corrosion 1996, 52, 169-176. [CrossRef]

24. Li, Y.; Su, L.; Shou, C.; Yu, C.; Deng, J.; Fang, Y. Surface-Enhanced Molecular Spectroscopy (SEMS) Based on Perfect-Absorber Metamaterials in the Mid-Infrared. Sci. Rep. 2013, 3, 1-8. [CrossRef] [PubMed]

25. Kim, J.; Lee, J.; Lee, K.; Yi, S. Enhanced Characteristics of Nondispersive Infrared $\mathrm{CO}_{2}$ Gas Sensor by Deposition of Hydrophobic Thin Film. In Proceedings of the Eurosensors 2017, Paris, France, 3-6 September 2017; Volume 1, pp. 1-5.

26. Mendes, L.B.; Ogink, N.W.M.; Van Dooren, H.J.C.; Mosquera, J.; Gerais, M. NDIR Gas Sensor for Spatial Monitoring of Carbon Dioxide Concentrations in Naturally Ventilated Livestock Buildings. Sensors 2015, 15, 11239-11257. [CrossRef] [PubMed]

27. Gordon, I.E.; Rothman, L.S.; Hill, C.; Kochanov, R.V.; Tan, Y.; Bernath, P.F.; Birk, M.; Boudon, V.; Campargue, A.; Chance, K.V.; et al. The HITRAN2016 Molecular Spectroscopic Database. J. Quant. Spectrosc. Radiat. Transf. 2017, 203, 3-69. [CrossRef]

28. NIST Chemistry WebBook. 2018. Available online: https://webbook.nist.gov/chemistry/ (accessed on 28 June 2018).

29. Eisberg, R.; Resnick, R.; Brown, J. Quantum Physics of Atoms, Molecules, Solids, Nuclei, and Particles; Wiley John \& Sons: Hoboken, NJ, USA, 1986; Volume 39.

30. Kim, J.; Yi, S. Effects of Temperature and Humidity on NDIR $\mathrm{CO}_{2}$ Gas Sensor. J. Sens. Sci. Technol. 2017, 26, 179-185. [CrossRef]

31. Ranganath, G.S. Black-Body Radiation. Resonance 2008, 13, 115-133. [CrossRef]

32. Ma, L.; Yang, J.; Nie, J. Two Forms of Wien's Displacement Law. Education 2009, 3, 566-568. 
33. Maikala, R.V. Modified Beer's Law-Historical Perspectives and Relevance in near-Infrared Monitoring of Optical Properties of Human Tissue. Int. J. Ind. Ergon. 2010, 40, 125-134. [CrossRef]

34. Sieber, I.; Eggert, H.; Suphan, K.; Oliver, N. Optical Modeling of the Analytical Chamber of an IR Gas Sensor. Lamp 2001, 4408, 272-282.

35. Viola, R. High-Luminosity Multipass Cell for Infrared Imaging Spectroscopy. Appl. Opt. 2006, 45, $2805-2809$. [CrossRef] [PubMed]

36. Martin, H. Gas Sensor. Swedish Patent SE 510549, 13 November 1995.

37. Zhang, Y.; Gao, W.; Song, Z.; An, Y.; Li, L.; Song, Z.; Yu, W.W.; Wang, Y. Design of a Novel Gas Sensor Structure Based on Mid-Infrared Absorption Spectrum. Sens. Actuators B Chem. 2010, 147, 5-9. [CrossRef]

38. Zheng, C.T.; Ye, W.L.; Li, G.L.; Yu, X.; Zhao, C.X.; Song, Z.W.; Wang, Y.D. Performance Enhancement of a Mid-Infrared $\mathrm{CH}_{4}$ detection Sensor by Optimizing an Asymmetric Ellipsoid Gas-Cell and Reducing Voltage-Fluctuation: Theory, Design and Experiment. Sens. Actuators B Chem. 2011, 160, 389-398. [CrossRef]

39. Park, J.; Min, N.; Kweon, S.; Yi, S. Novel NDIR $\mathrm{CO}_{2}$ sensor with two concave mirrors. In Proceedings of the Asia-Pacific Conference of Transducers and Micro-Nano Technology (APCOT) 2006, Singapore, 25-28 June 2006; p. 137.

40. Jang, S.; Chung, S.; Yi, S. Characteristics of an Optical Waveguide with Two Identical Elliptical Structures. J. Korean Inst. Gas. 2014, 18, 48-54. [CrossRef]

41. Kim, J.; Yi, S. Properties of Non-Dispersive Infrared Ethanol Gas Sensors according to the Irradiation Energy. J. Sens. Sci. Technol. 2017, 26, 168-172. [CrossRef]

(C) 2018 by the authors. Licensee MDPI, Basel, Switzerland. This article is an open access article distributed under the terms and conditions of the Creative Commons Attribution (CC BY) license (http://creativecommons.org/licenses/by/4.0/). 